\title{
Teaching Paradigm Reform Based on Semi-Flipped Classroom and a Preliminary Practice
}

\author{
Lingjian Ye, Xiushui Ma, Weihong Zhong \\ Ningbo Institute of Technology, Zhejiang University \\ Ningbo 315100, Zhejiang, China \\ ljye@nit.net.cn, mxsh63@aliyun.com, zwh@nit.net.cn
}

\author{
Hongwei Guan \\ Ningbo Dahongying University \\ 315175, Ningbo, Zhejiang, China \\ ghw_nit@126.com
}

\begin{abstract}
The flipped classroom paradigm brought up innovative reforms into the teaching field and has been prevalent globally in the recent years. However, the success of a flipped classroom relies on various supporting elements, which cannot be generally guaranteed in a particular college education. To release the strictness so as to enhance the college education, this study proposes a semi-flipped classroom paradigm which integrates the models of the traditional teaching paradigm and the flipped classroom. The new teaching pattern is designed and the fundamental elements of a semi-flipped classroom are elaborated in detail. In a scientific way, the new teaching paradigm absorbs both advantages of the two sides, hence more suitable for the college education. Preliminary practice has been carried out in a college course, which showed a great potential of the new paradigm.
\end{abstract}

Keywords-Flipped classroom; Hybrid paradigm; Teaching practice; College education

\section{INTRODUCTION}

The newly emerged flipped classroom paradigm brought up innovative reforms in the teaching field and has been prevalent globally in the recent years. Around the beginning of this century, the Harvard professor, Eric Mazur developed the concept he called peer instruction, which is an instructional strategy that involves flipped teaching [1,2]. Later, Salman Khan made a great effort pushing forward the possibility of flipped classroom by recording lesson videos and provided them to the public free of service. He later founded the Khan Academy based on the flipped pattern and promoted the teaching model significantly [3,4]. The core feature of the flipped classroom is that it reverses the traditional learning environment. That is, it moves the instructional teaching content (often online videos), outside of the classroom, and learner activities (often the after-class homework), into the classroom.

In a flipped classroom, the content can be delivered outside of the classroom in a variety of forms, typically via video lessons, prepared by either teachers or the third parties, such as the well-known Khan Academy mentioned above. Based on the gained preliminary knowledge, the in-class activities can be focused on deeper discussions and personalized guidance by the mentor, in which form the classroom is more efficiently utilized. The flipped classroom converts the teacher-centered activity into the learner-centered model, which makes the students feel highly engaged and iterative with the teacher.

This work was supported by Ningbo Education Planning Project (2016YGH032)
Massive successful applications of the flipped classroom were reported along different level educations [5-8], including cases from middle school to colleges/universities. Furthermore, the integrations of flipped classroom and other educational approaches are also promoted, e.g. the flipped adaptive learning [9] and so forth.

Though widely beneficial, the flipped classroom itself has limitations and be criticized in several ways [10-12]. For example, not all students are self-disciplined in their own home learning environments. Without after-class commitments, the students may fall rapidly behind their peers. Additionally, teachers also need significant preparation time outside of the traditional teaching responsibilities, which is particularly an issue for college teachers who engaged themselves in the academic researches. Quoting the whitepaper by Hamdan et al. [13], the success of flipped learning model depends on four fundamental elements: Flexible Environment, Learning Culture, Intentional Content and Professional Educator, which are not always satisfied in a particular environment. For example, in the college where the authors work, the students are less initiative in a highly disturbed learning atmosphere, the learning culture is unlikely to transformed and hold firmly. In this case, the advantage of the flipped classroom over the traditional teaching model is questionable.

Various investigations and observations in the college education motivate us to redesign the flipped classroom model such that it can be adaptive to the particular environment, in which background we propose the semi-flipped classroom paradigm. More detailed analysis and supporting elements are given in the sequel.

\section{THE SEMI-FLIPPED CLASSROOM}

\section{A. The flipped classroom}

The conventional teaching model is typically composed of three elements, namely, the "teach, practice and review". The teacher is the core role whereas the students are in a passive position. The process is often lack of discussions and the review feedback is highly delayed. However, in a flipped classroom model, the three key elements are "learn, test and level-up study", which transforms the students into the positive position. Preliminary knowledge is acquired before the classroom through various possible forms, typically online videos. Teachers in the classroom answer questions gathered from students and carried out deep and personalized guidance, 
such as tests and innovative discussions. The process is highly interactive and the feedback is timely. The structural diagrams of the two teaching model are compared in Fig.1.

\section{Traditional teaching model \\ Flipped classroom}

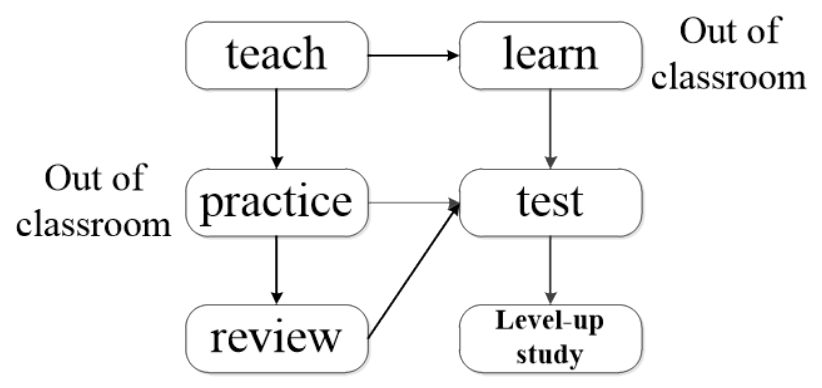

Fig. 1. Structural diagrams of the two teaching models

\section{B. Motivations}

However, the success of a flipped classroom depends on a wide range of supporting elements. For example, Hamdan et al. [13] think the flipped learning model depends on four fundamental elements: Flexible Environment, Learning Culture, Intentional Content and Professional Educator. Basically, the flipped classroom requires cooperation from three parties: the environment, students and the teacher. Over years of college education and with earlier efforts to promote the flipped classroom model, we found that as college teachers ourselves, some elements are difficult to fulfill requirements of a flipped classroom, especially for those associated with the environment and students. Some of the main obstacles are described as follows:

- College students usually need to attend a dozen of courses in one semester. Since the flipped classroom requires massive after-class effort and time for selflearning, it is unrealistic to put forward flipped classroom for more than a few courses at the same time.

- Most courses are still conducted in the regular way and most of them last only one semester. Since the students have been long get used to the regular teaching model, it is unreasonable for them to learn the pattern of a flipped classroom in one semester and then discard the pattern once the course is finished.

- Not all college students are strongly initiative in courses, particularly when the college atmosphere is highly disturbed, such as the engagements in social activities and entertainments. Investigations and informal conversations with student representatives have been previously carried out. More than 90\% students conveyed that they would not spend more than 3 hours on one course per week, and more than $50 \%$ would not accept even 1 hour. Since in a flipped classroom model, massive time should be spent on their own outside the classroom, it is unlikely to succeed using the standard flipped classroom.

\section{The semi-flipped classroom model}

Motivated by the above reasons, we propose a new semiflipped classroom model, which, on one hand, absorbs the advantages of the flipped classroom model and on the other, makes the paradigm adaptive to customized college education. Compared to the full flipped model, we preserve in part the conventional teach module. The structure of the semi-flipped classroom model is illustrated in Fig.2.

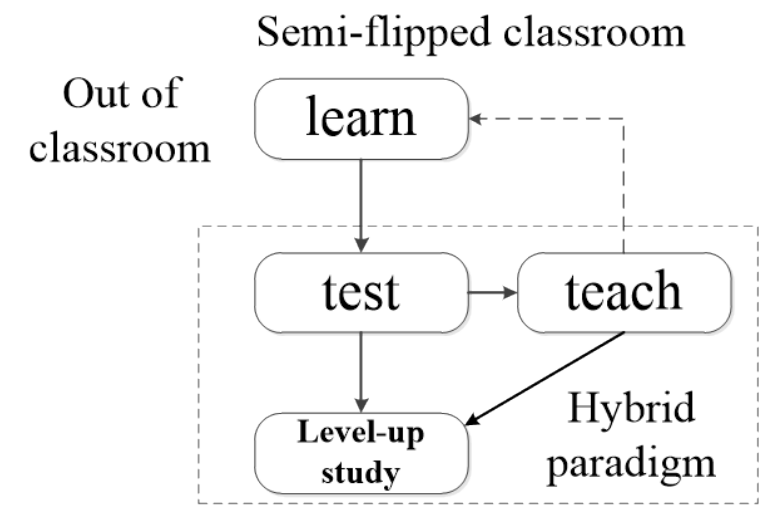

Fig. 2. Structural diagram of the semi-flipped classroom model

To elaborate the differences of the new model, one notes that the within-classroom activities are composed of both the test and teach which combines the two different teaching models. The test block is conducted in the flipped classroom style and the other is conducted in the conventional style, each of which is about 50 percent occupation, which we call the module a hybrid paradigm.

Outside the classroom, the students still need to learn as in the flipped classroom, but the content and strength should be different, as explained in the following.

\section{Key elements in the semi-flipped classroom}

The key elements of a semi-flipped classroom are as follows:

- Elegant organization of within-classroom activities. As has been mentioned, the activities are composed of the test and teaching, which are from the two different teaching models, respectively. Although the time for each part has been halved, one teaching unit in the college education is often more than one and a half hours. Therefore, both of them can still be up to $45 \mathrm{~min}$ in the new teaching model.

- Concise and highly condensed teaching syllabus. The course syllabus has to be adapted due to the new course paradigm with the teaching time reduced. Basically, the syllabus should be concise and highly condensed. We propose to design the course syllabus with conjunction of the major's objectives and further keep consistence with other following-up courses. Nonetheless, the key goal is to let student know the methodologies and ways of dealing with practice problems. The syllabus should reflect this point. 
- Well prepared within-class tests and quiz. The tests and quiz should be prepared along the syllabus to highlight the main knowledge points. The problems should be enlightening and initiative. During the preliminary practice, we found that the difficulty extents for the problems are of critic importance.

- Suitable after-class material. The after-class materials are also not limited to a particular form, as long as they are suitable to serve the main objective. It should be stressed that substantial materials are not necessary due to the limited after-class effort mentioned above. Experiences indicate that it is greatly helpful that the teachers raise particular questions in the teach part inside the classroom, such that students can purposely search answers going through the after-class materials.

\section{E. Advantages}

In some sense, the semi-flipped classroom is a compromised version of the full flipped classroom, which has encountered difficulty in the college courses. The semi-flipped classroom is proposed to overcome the limitations, in our views, the advantages of the semi-flipped model are in multifolds:

(1) The within-class activities are also interactive and highly efficient, which allow for the level-up study, the same as in the classic flipped classroom;

(2) The teach module convey knowledge points in a conventional way, which is familiar by the students and easily accepted;

(3) The long class unit (in the college) is divided into different activities, which are not likely to let student feel bored due to the role transition. Otherwise, the learners easily lose their patience in less than one hour, according to our experiences;

(4) The new concise and condensed teaching syllabus is more suitable for the college education, especially for those practice-oriented colleges.

\section{PRELIMINARY PRACTICE IN THE “PROCESS PRINCIPLE”} COURSE

\section{A. Backgrounds}

"Process Principle"(PP) is an elementary course for the major: Automation (direction: process control). It concerns with chemical processes and how chemical materials are transported, separated and purified. The aim of this course is to help students build a conception of the industrial backgrounds of process control and learn basic methodologies, based on which later automation courses are easily understood. However, the course itself is very difficult and abundant in content. Previously, the course was conducted by teachers from the Department of Chemical Engineering and delivery knowledge in a different way. Until recent years, the course was taken over by the authors majoring Process Control. In the latest teaching plans, the whole course contains 16 teaching units, each of which is $90 \mathrm{~min}$.

\section{B. Customized Semi-flipped classroom model}

The design of semi-flipped classroom model customized to the PP course is carried out in several aspects. Note that the process is gradually taken place over several years of teaching and until recently we formulated the concept of semi-flipped classroom. And it is also in another teaching project, the "fourdimensional integration"

a) Syllabus redesign. We redesigned a highly condensed syllabus of PP, which is mainly composed of three chapters: the hydrodynamics of flow, the heat transfer and the mass transfer, each of which occupies roughly 5 teaching units. These three chapters are core ideas of the PP course based on our experience, which contain representative knowledge points and most important mathematical methodologies. The focus is on the most fundamental points, but the selected ones are still integral to form a complete picture of the course. The main points of the syllabus are shown in TABLE I.

TABLE I. SYLLABUS OF PP

\begin{tabular}{|c|l|}
\hline Chapter & \multicolumn{1}{|c|}{ Knowledge points } \\
\hline $\begin{array}{c}\text { Chapter 1. } \\
\text { hydrodynamics }\end{array}$ & $\begin{array}{l}\text { Fluid statics equations, N-S equation, physical } \\
\text { properties of flow and calculation methods, Bernoulli } \\
\text { equation and applications, Bernoulli equation, Reynolds } \\
\text { number, friction calculation, flow meters }\end{array}$ \\
\hline $\begin{array}{c}\text { Principle of heat conduction, heat transfer force and } \\
\text { Chapter 2. heat } \\
\text { transfer }\end{array}$ & $\begin{array}{l}\text { resistance, heat transfer rate equation, transfer } \\
\text { coefficient, energy balance, averge temperature } \\
\text { calculation, the design and operation problems of heat } \\
\text { exchangers, LMTD method and } \varepsilon-N T U \text { method }\end{array}$ \\
\hline $\begin{array}{c}\text { Relative volatility, vapor-liquid phase equilibrium, } \\
\text { principle of distillation, calculation of distillation, } \\
\text { equaiton of operation lines(distillation line, stripping } \\
\text { mass transfer } 3 .\end{array}$ & $\begin{array}{l}\text { line, q-line), calculation of paramter q, theoritical tray } \\
\text { number, minimal reflux ratio, the design and operation } \\
\text { problems of distillation columns }\end{array}$ \\
\hline
\end{tabular}

b) Organization of within-classroom activities. As has been explained, in the semi-flipped classroom, the activities are composed of test and teaching. In the teaching part, we proceed in the regular way, except that the knowledge points are redesigned in the new syllabus. Furthermore, at the end of the teaching, illuminating questions will be raised, where the answers can be found in the after-class materials. In the test part, we prepared more than 100 numerical problems came from industrial applications. These problems are selected and chosen based on experiences over years of teaching and updated every year. The problems are tested within the classroom, during which discussions are encouraged among grouped students, but plagiarism is strictly forbidden.

c) After-class materials. After-class materials include appropriate textbooks, electronic slides, flash, and so forth. These materials are also associated with the syllabus. In the materials, we highlighted the importance of industrial applications and real process control projects. Innovative questions and knowledge are embedded in these materials. For now, self-made videos are still under constructions.

\section{Results}

The preliminary practice of the semi-flipped classroom is carried out experimentally. We collect feedbacks from student 
representatives, which showed the following main points: (1) the syllabus is concise and relatively easy to grab the key knowledge points; (2) the hybrid teaching paradigm is interesting and fresh new to the students. With the new arrangements, most of the students won't bored and interested in taking more courses like this. (3) the after-class materials take acceptable time.

In general, we observed that the average grade in the final exams has been improved by about $12 \%$ during the experimental practice. Furthermore, many students showed strong interests in taking other courses, as well as scientific research projects, in charge of the same teachers.

\section{CONCLUSIONS}

In this study, we proposed a new semi-flipped classroom teaching paradigm, which is to adapt the popular flipped classroom teaching paradigm suitable for college education. In the semi-flipped classroom, we combine the activities in a flipped classroom and regular teaching in the conventional way. Due to these adaptions, the syllabus, as well as other contents, needs to be changed accordingly. However, with appropriate designs, the semi-flipped classroom can be advantageous over both of the conventional teaching and the flipped classroom. That is, the advantages of the both sides are absorbed in the new pattern in a scientific way. In the preliminary practice of the semi-flipped classroom to the "process principle" course, we obtained improved results and positive feedbacks from the students. In the future, the framework of semi-flipped classroom teaching paradigm will be further consolidated and improved. The after-class materials of the process principle course will also be supplemented, such as the online videos.

\section{REFERENCES}

[1] E. Mazur, "Peer Instruction: A User's Manual Series in Educational Innovation. Prentice Hall, Upper Saddle River, NJ, 1997.

[2] C. Crouch and E. Mazur, "Peer Instruction: Ten Years of Experience and Results”, Am. J. Phys., 2001, v69, 970-977.

[3] C. Thompson, "How Khan Academy is Changing the Rules of Education", Wired Magazine, 15 July, 2011.

[4] S. D. Sparks, "Lectures Are Homework in Schools Following Khan Academy Lead”, 28 Sep, 2011.

[5] J. Bergmann and A. Sams, "Flip your classroom: reach every student in every class every day”. Washington, DC: International Society for Technology in Education, 2012.

[6] T. Rosenberg, "Turning Education Upside Down". New York Times. Retrieved 2013-10-11.

[7] S. Muhammed, F. Kurban and Caroline, "The Flipped Approach to Higher Education: Designing Universities for Today's Knowledge Economies and Societies”. UK: Emerald. 2 August, 2016.

[8] N. Johnston and T. Karafotias, "Flipping the Classroom to Meet the Diverse Learning Needs of Library and Information Studies (LIS) Students”. Journal Of Education For Library \& Information Science, 57(3), 226-238, 2016.

[9] K. Kaw, M. Besterfield-Sacre, A. Scott, R. Pendyala and Y. Lou, "Improving and Assessing Student Learning in an Inverted STEM Classroom Setting”, https://nsf.gov/awardsearch/showAward?AWD_ID $=1322586$.

[10] L. Nielsen, "Five reasons I'm not flipping over the flipped classroom", 2012

[11] J. F. Strayer, J. F. "How learning in an inverted classroom influences cooperation, innovation and task orientation”. Learning Environments Research, 15(2), 171-193. 2012.

[12] N. Lents and O. Cifuentes. "Web-based learning enhancements: Video lectures through voice-over PowerPoint in a Majors-level Biology course". Journal of College Science Teaching, 39 (2): 38-46, 2009.

[13] N. Hamdan, P. McKnight, K. McKnight, “The flipped learning model: A white paper based on the literature review titled a review of flipped learning”. Flipped Learning Network/Pearson/George Mason University, 2013 